\title{
Constraints on micro-Raman strain metrology for highly doped strained Si materials
}

\author{
L. O’Reilly, ${ }^{1, a)}$ K. Horan, ${ }^{1}$ P. J. McNally, ${ }^{1}$ N. S. Bennett ${ }^{2}$ N. E. B. Cowern, ${ }^{2}$ A. Lankinen, ${ }^{3}$ \\ B. J. Sealy, ${ }^{4}$ R. M. Gwilliam, ${ }^{4}$ T. C. Q. Noakes, ${ }^{5}$ and P. Bailey ${ }^{5}$ \\ ${ }^{1}$ Nanomaterials Processing Laboratory, Research Institute for Networks and Communications Engineering \\ (RINCE), School of Electronic Engineering, Dublin City University, Dublin 9, Ireland \\ ${ }^{2}$ School of Electrical, Electronic and Computer Engineering, University of Newcastle, Newcastle upon Tyne, \\ NE1 7RU, United Kingdom \\ ${ }^{3}$ Micro and Nanosciences, Micronova, Helsinki University of Technology, P.O. Box 3500, \\ FIN-02015 TKK, Finland \\ ${ }_{5}^{4}$ Surrey Ion Beam Centre, University of Surrey, Guildford GU2 7XH, United Kingdom \\ ${ }^{5}$ Medium Energy Ion Scattering Facility, Daresbury Laboratory, Daresbury, Warrington WA4 4AD, United \\ Kingdom
}

(Received 22 February 2008; accepted 20 May 2008; published online 12 June 2008)

\begin{abstract}
Ultraviolet (UV), low penetration depth, micro-Raman spectroscopy, and high-resolution x-ray diffraction (HRXRD) are utilized as complementary, independent stress characterization tools for a range of strained Si samples doped by low energy (2 keV) Sb ion implantation. Following dopant implantation, good agreement is found between the magnitudes of strain measured by the two techniques. However, following dopant activation by annealing, strain relaxation is detected by HRXRD but not by micro-Raman. This discrepancy mainly arises from an anomalous redshift in the Si Raman peak position originating from the high levels of doping achieved in the samples. This has serious implications for the use of micro-Raman spectroscopy for strain characterization of highly doped strained Si complementary metal-oxide semiconductor devices and structures therein. We find a direct correlation between the Si Raman shift and peak carrier concentration measured by the differential Hall technique, which indicates that UV micro-Raman may become a useful tool for nondestructive dopant characterization for ultrashallow junctions in these Si-based materials. (C) 2008 American Institute of Physics. [DOI: 10.1063/1.2942392]
\end{abstract}

The enhancement of carrier mobilities through the introduction of strain in the channel region of metal-oxide semiconductor field effect transistors is an important option in order to maintain historical complementary metal-oxide semiconductor (CMOS) performance trends. ${ }^{1}$ The production of ultrashallow junctions for the source/drain extension region using low energy ion implantation will be required for future CMOS devices. Biaxial tensile strain reduces the sheet resistance $\left(R_{S}\right)$ of highly doped $n$ type layers created by As or $\mathrm{Sb}$ implantation. The effect can be stronger for $\mathrm{Sb}$, when $R_{s}$ lowering results not only from strain enhanced mobility, but also from an improvement in Sb metastable solubility in the presence of strain. ${ }^{2}$ This makes Sb an interesting alternative to As for ultrashallow junctions in strain-engineered CMOS devices. Since an early study by Anastassakis et al., ${ }^{3}$ Raman scattering has been widely used as a strain characterization technique in the semiconductor industry ${ }^{4-6}$ and is often utilized for strain metrology in strained Si films. ${ }^{7,8}$ Our results show that there are practical difficulties associated with the use of micro-Raman as a strain characterization technique for highly doped ultrashallow junction Si CMOS device structures.

Experiments were performed on two types of tensilestrained $\mathrm{Si}$ wafers each grown on $\mathrm{Si}_{1-x} \mathrm{Ge}_{x}$ relaxed buffer layers with $x=0.2$ and $x=0.17$, and strained Si thicknesses of 43 and $17.5 \mathrm{~nm}$, respectively. Each wafer was implanted with a $2 \mathrm{keV} \mathrm{Sb}$ ion dose of between $1 \times 10^{14}$ and $1 \times 10^{15} \mathrm{~cm}^{-2}$ creating a junction at a depth of around $10 \mathrm{~nm}$.

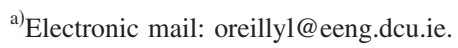

Nominally unstrained control samples were prepared using conventional $p$-type $\mathrm{Si}$ wafers for comparison. The implanted dose was confirmed by medium-energy ion scattering (MEIS). Dopant activation was achieved by rapid thermal annealing (RTA) wafer pieces for $10 \mathrm{~s}$ in $\mathrm{N}_{2}$ in the range of $600-800{ }^{\circ} \mathrm{C}$. Room temperature micro-Raman measurements were performed with a Jobin Yvon LabRam HR800 system in backscattering geometry using a $325 \mathrm{~nm} \mathrm{He}-\mathrm{Cd}$ UV laser excitation with a spot size of approximately $1 \mu \mathrm{m}$. High-resolution x-ray diffraction (HRXRD) measurements were performed on a Philips X'Pert Pro diffractometer. Reciprocal space maps were collected with a three-bounce germanium analyzer crystal placed before the detector, which narrows the acceptance angle to approximately 12 arc sec. Peak carrier concentrations were measured by the differential Hall profiling technique. ${ }^{9}$

Due to the limited UV laser penetration into the samples (9 $\mathrm{nm})$, the Raman spectra of both the reference unstrained $\mathrm{Si}$ bulk and the strained Si samples are dominated by the signal of the degenerate transverse optical $\mathrm{Si}-\mathrm{Si}$ phonon mode. For the bulk crystalline Si wafer, this Si peak appears at a Raman shift of $520 \mathrm{~cm}^{-1}$. A redshift (lower wavenumber) in the $\mathrm{Si}-\mathrm{Si}$ Raman peak position of the strained $\mathrm{Si}$ samples compared to that of the reference unstrained $\mathrm{Si}$ sample indicates that a tensile stress is present in the Si cap layer. ${ }^{4}$ The total peak shift of the Si-Si Raman mode following $\mathrm{Sb}$ ion implantation and RTA, relative to the reference unstrained Si peak position is shown in Fig. 1 for each set of strained Si samples. In principle, strain relaxation would be detected by a blueshift (higher wavenumber) of the Si Ra- 


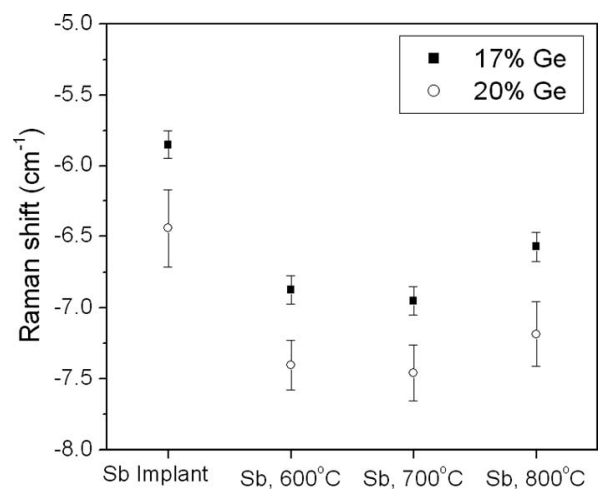

FIG. 1. Raman redshift relative to unstrained $\mathrm{Si}$ for $\mathrm{Sb}$-implanted strained $\mathrm{Si}$ samples grown on relaxed buffer layers with $17 \%$ or $20 \%$ Ge. Samples were measured as implanted and after annealing at 600,700 or $800{ }^{\circ} \mathrm{C}$ for $10 \mathrm{~s}$.

man peak. However, in Fig. 1 we clearly see a redshift of the $\mathrm{Si}-\mathrm{Si}$ phonon mode peak position relative to the implanted case, at annealing temperatures of 600 and $700^{\circ} \mathrm{C}$. At $800{ }^{\circ} \mathrm{C}$ a net redshift is still observed although it is less than for the lower annealing temperatures. Conventionally, this redshift of the Si-Si Raman phonon mode peak position observed in all samples after RTA would be attributed to an increase in tensile strain. ${ }^{4}$ Reciprocal space maps were obtained using HRXRD in order to gain strain information for the samples independently from micro-Raman spectroscopy. The 422 reflection in glancing-incidence geometry was measured according to the procedure reported by Erdtmann and Langdo. ${ }^{10}$ Our HRXRD measurements have shown that slight strain relaxation occurs during RTA at all temperatures in our range of interest. This is consistent with work recently published on misfit strain relaxation in strained $\mathrm{Si}^{11}{ }^{11}$ We propose that a further contribution to the Raman redshift arising from the high doping levels in the sample obscures any Raman blueshift due to strain relaxation, accounting for this disagreement between HRXRD and micro-Raman techniques.

In Figs. 2(a) and 2(b), the absolute Raman redshift relative to the Si Raman peak position of the as-implanted sample is plotted alongside the peak donor carrier concentrations measured as a function of dose for strained and unstrained samples. A Hall scattering correction factor of 1.0, estimated from MEIS was applied to all the $\mathrm{Sb}$ doped samples. For all doses, the experimental points are for the samples annealed at $700{ }^{\circ} \mathrm{C}$ for which we obtained our op-

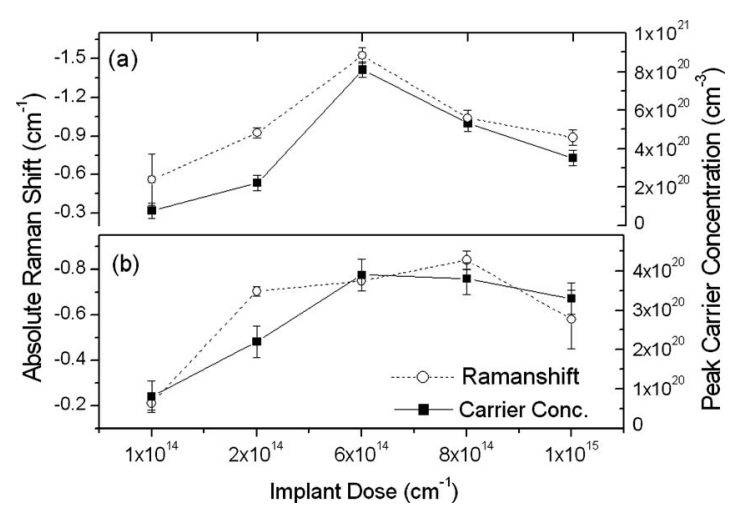

FIG. 2. Peak carrier concentration and absolute Raman redshift as a function of implant dose for (a) $2 \mathrm{keV} \mathrm{Sb-implanted} \mathrm{strained} \mathrm{Si}$ after $10 \mathrm{~s}$ RTA at

$700{ }^{\circ} \mathrm{C}$ and (b) $2 \mathrm{keV} \mathrm{Sb-implanted} \mathrm{bulk} \mathrm{Si} \mathrm{after} 10 \mathrm{~s} \mathrm{RTA}$ at $700{ }^{\circ} \mathrm{C}$. theory developed for bulk Si may also be applied to strained
Downloaded 30 Mar 2009 to 131.227 .178 .132 . Redistribution subject to AIP license or copyright; see http://apl.aip.org/apl/copyright.jsp timum electrical results. In the strained case, we find the maximum level of $\mathrm{Sb}$ activation at a dose of $6 \times 10^{14} \mathrm{~cm}^{-2}$. The largest absolute Raman redshift of $1.53 \pm 0.06 \mathrm{~cm}^{-1}$ is also detected for this sample. The Sb deactivation at higher doses, likely due to the formation of Sb clusters or precipitates, results in a reduction in the peak carrier concentration that is mirrored by a decrease in the absolute Raman redshift. In the unstrained samples [Fig. 2(b)] the peak carrier concentrations and values of the absolute Raman redshift are again seen to follow similar trends. This supports our assertion that our measured Raman shift for these samples is correlated to high doping levels and not to strain. In the unstrained case we do not have a sharp maximum in the mid-dose range, which we believe is caused in the strained case by an increase in the solubility of $\mathrm{Sb}$ with strain present. A detailed discussion of our electrical data has been published elsewhere. ${ }^{1,12}$ By way of example, if we apply conventional Si Raman strain theory ${ }^{4}$ to the maximum Raman shift of $-1.5 \mathrm{~cm}^{-1}$, such a shift would indicate an increase in tensile stress by $\sim 375 \mathrm{MPa}$, which is clearly at odds with HRXRD reciprocal space map measurements. In any case the introduction of large $\mathrm{Sb}$ atoms should lower the tension in the upper strained $\mathrm{Si}$ layer. From the minimum values of peak carrier concentration and their corresponding Raman redshifts, we propose that the use of micro-Raman spectroscopy to directly measure strain in $n$-doped samples is limited to samples with electron carrier concentrations $<5 \times 10^{19} \mathrm{~cm}^{-3}$. For higher net doping levels, a correction must be applied by utilizing either electrical or strain data independently obtained from Raman spectroscopy. Thus, the combination of Raman spectroscopy with either the differential Hall technique or HRXRD yields information on both strain and doping levels in these samples. For nominally undoped samples, we find micro-Raman spectroscopy to be a fast and effective method for direct strain characterization with high spatial resolution.

An early study ${ }^{13}$ on the dependence of the first-order Raman frequency of Si on carrier concentration $N\left(\mathrm{~cm}^{-3}\right)$ found that doping alters the lattice deformation potential of $\mathrm{Si}$, resulting in an effective softening of the lattice giving lower phonon vibrational frequencies (Raman redshifts) at higher carrier concentrations. This effect has also been experimentally observed in nanocrystalline ${ }^{14}$ and polycrystalline ${ }^{15} \mathrm{Si}$ films. The following expression was developed in the case of $n$-type bulk $\mathrm{Si}^{13}$

$$
10^{3}(\delta \omega / \omega)=-0.025 X\left(1+0.02 X^{2 / 3}\right), \quad X=10^{-19} \mathrm{~N},
$$

where $\omega$ is the wavenumber of the doped Si Raman peak and in our case $\delta \omega$ is the absolute Raman shift relative to the implanted unstrained or strained Si peak position, respectively. This absolute Raman shift for bulk and strained $\mathrm{Si}$ samples doped by $\mathrm{Sb}$ ion implantation during $10 \mathrm{~s}$ RTA at $700{ }^{\circ} \mathrm{C}$ is plotted in Fig. 3 as a function of peak carrier concentration as measured by the differential Hall technique. Qualitatively, the data follow the theory in Ref. 13-the absolute value of the relative Raman Si peak becoming more redshifted as a function of increasing carrier concentration. This theory is subject to adjustment due to an error in the value of the deformation potential of the Si conduction band used by the authors, ${ }^{16}$ which may account for some of the difference between the theory and our experimental data. A linear fit of bulk and strained Si data points indicates that the theory developed for bulk Si may also be applied to strained to AIP license or copyright; see http://apl.aip.org/apl/copyright.jsp 


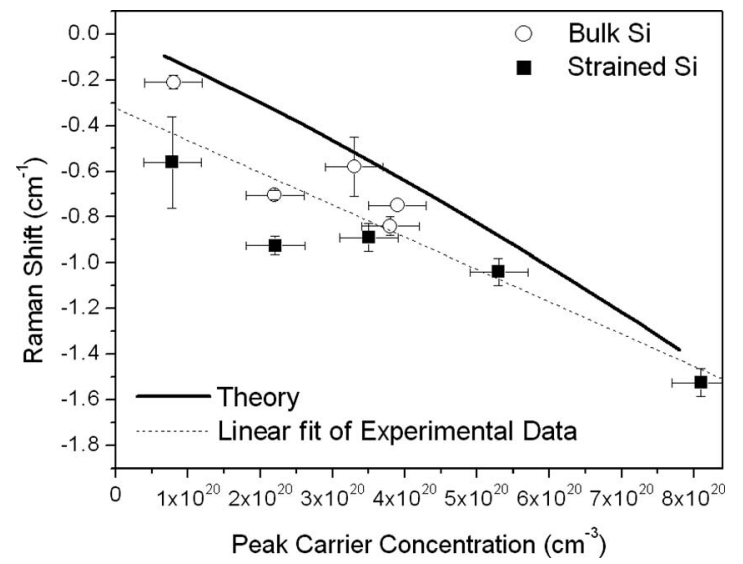

FIG. 3. Absolute Raman shifts as a function of peak carrier concentration for $2 \mathrm{keV} \mathrm{Sb-implanted} \mathrm{bulk} \mathrm{and} \mathrm{strained} \mathrm{Si} \mathrm{after} 10 \mathrm{~s}$ RTA at $700{ }^{\circ} \mathrm{C}$. We include the theory of Ref. 13 as given by Eq. (1). A linear regression fit of the bulk and strained $\mathrm{Si}$ experimental data points yielded an $R^{2}$ value of 0.89 .

$\mathrm{Si}$. However, Raman frequencies in strained $\mathrm{Si}$ are slightly differently affected by the carrier concentration compared to bulk $\mathrm{Si}$ as on average we observe larger Raman redshifts for strained samples compared to bulk samples with similar doping levels. This may be related to the preferential occupation of the two out-of-plane conduction band valleys with respect to the four in-plane valleys in strained $\mathrm{Si}^{17}{ }^{17}$ However, at this stage of our investigations, we do not rule out additional sources of Raman redshift in the strained Si samples.

In summary, a direct correlation has been found between the relative frequency of the Si Raman peak and the electron carrier concentration. Caution must therefore be exercised when using micro-Raman spectroscopy to extract strain information from samples with electron carrier concentrations $>5 \times 10^{19} \mathrm{~cm}^{-3}$. For such cases, we recommend the use of an independent strain measurement technique such as HRXRD. However, this Raman effect could prove to be a useful nondestructive means of extracting carrier concentra- tion information from very thin, highly doped strained Si layers desirable for future CMOS devices.

Science Foundation Ireland is gratefully acknowledged for funding this project under the Investigator Programme Grant Scheme Project 05/IN/I656. The authors would like to thank R. Harper of IQE Silicon Compounds, Ltd., and H. Radamson of KTH, Sweden for providing the strained silicon substrates. Authors L.O.R and K.H equally contributed as first authors to the work outlined in this manuscript.

${ }^{1}$ D. A. Antoniadis, I. Aberg, C. Ní Chléirigh, O. M. Nayfeh, A. Khakifirooz, and J. L. Hoyt, IBM J. Res. Dev. 50, 363 (2006).

${ }^{2}$ N. S. Bennett, N. E. B. Cowern, A. J. Smith, R. M. Gwilliam, B. J. Sealy, L. O'Reilly, P. J. McNally, G. Cooke, and H. Kheyrandish, Appl. Phys. Lett. 89, 182122 (2006).

${ }^{3}$ E. Anastassakis, A. Pinczuk, E. Burstein, F. H. Pollack, and M. Cardona, Solid State Commun. 8, 133 (1970).

${ }^{4}$ I. De Wolf, H. E. Maes, and S. K. Jones, J. Appl. Phys. 79, 7148 (1996).

${ }^{5}$ K. F. Dombrowski, I. De Wolf, B. Dietrich, Appl. Phys. Lett. 75, 2450 (1999).

${ }^{6}$ C. Himcinschi, I. Radu, R. Singh, W. Erfurth, A. P. Milenin, M. Reiche, S. H. Christiansen, and U. Gösele, Mater. Sci. Eng., B 135, 184 (2006).

${ }^{7}$ J. Schmidt, G. Vogg, F. Bensch, S. Kreuzer, P. Ramm, S. Zollner, R. Liu, and P. Wennekers, Mater. Sci. Semicond. Process. 8, 267 (2005).

${ }^{8}$ B. Jin, X. Wang, J. Chen, X. Cheng, and Z. Chen, Appl. Phys. Lett. 87, 051921 (2005)

${ }^{9}$ N. S. Bennett, A. J. Smith, B. Colombeau, R. Gwilliam, N. E. B. Cowern, and B. J. Sealy, Mater. Sci. Eng., B 124, 305 (2005).

${ }^{10}$ M. Erdtmann and T. A. Langdo, J. Mater. Sci.: Mater. Electron. 17, 137 (2006).

${ }^{11}$ J. Parsons, E. H. C. Parker, D. R. Leadley, T. J. Grasby, and A. D. Capewell, Appl. Phys. Lett. 91, 063127 (2007).

${ }^{12}$ N. S. Bennett, A. J. Smith, R. M. Gwilliam, R. P. Webb, B. J. Sealy, N. E. B. Cowern, L. O'Reilly, and P. J. McNally, J. Vac. Sci. Technol. B 26, 391 (2008).

${ }^{13}$ F. Cerdeira and M. Cardona, Phys. Rev. B 5, 1440 (1972).

${ }^{14}$ W. Wei, G. Xu, J. Wang, and T. Wang, Vacuum 81, 656 (2007).

${ }^{15}$ N. H. Nickel, P. Lengsfeld, and I. Sieber, Phys. Rev. B 61, 15558 (2000).

${ }^{16}$ M. Chandrasekhar, J. B. Renucci, and M. Cardona, Phys. Rev. B 17, 1623 (1978).

${ }^{17}$ M. L. Lee, E. A. Fitzgerald, M. T. Bulsara, M. T. Currie, and A. Lochtefeld, J. Appl. Phys. 97, 011101 (2005). 